\title{
Visual local and global processing in low-functioning deaf individuals with and without autism spectrum disorder
}

\author{
Jarymke Maljaars ${ }^{1}$, Ilse Noens ${ }^{2}$, Evert Scholte ${ }^{1}$, Roger Verpoorten ${ }^{3}$ \& Ina van Berckelaer-Onnes ${ }^{1}$ \\ ${ }^{1}$ Leiden University, the Netherlands; ${ }^{2}$ Katholieke Universiteit Leuven, Belgium, ${ }^{3}$ Koninklijke Kentalis, the Netherlands
}

\begin{abstract}
The ComFor study has indicated that individuals with intellectual disability (ID) and autism spectrum disorder (ASD) show enhanced visual local processing compared with individuals with ID only. Items of the ComFor with meaningless materials provided the best discrimination between the two samples. These results can be explained by the weak central coherence account. The main focus of the present study is to examine whether enhanced visual perception is also present in low-functioning deaf individuals with and without ASD compared with individuals with ID, and to evaluate the underlying cognitive style in deaf and hearing individuals with ASD. Different sorting tasks (selected from the ComFor) were administered from four subsamples: (1) individuals with ID $(n=68)$, (2) individuals with ID and ASD $(n=72)$, (3) individuals with ID and deafness $(n=22)$, and (4) individuals with ID, ASD and deafness $(n=15)$. Differences in performance on sorting tasks with meaningful and meaningless materials between the four subgroups were analyzed. Age and level of functioning were taken into account. Analyses of covariance revealed that results of deaf individuals with ID and ASD are in line with the results of hearing individuals with ID and ASD. Both groups showed enhanced visual perception, especially on meaningless sorting tasks, when compared with hearing individuals with ID, but not compared with deaf individuals with ID. In ASD either with or without deafness, enhanced visual perception for meaningless information can be understood within the framework of the central coherence theory, whereas in deafness, enhancement in visual perception might be due to a more generally enhanced visual perception as a result of auditory deprivation.
\end{abstract}

Keywords: autism spectrum disorder, central coherence, deafness, intellectual disability, visual perception

\section{Introduction}

The debate on cognitive theories explaining behavior and finding indications for possible brain dysfunctions in autism spectrum disorders (ASD) is still on-going. Frith (1989) introduced the concept of 'weak central coherence' (WCC). In ASD the drive for central coherence is rather weak, whereas in the normal cognitive system there is a natural tendency to integrate incoming stimuli and to interpret them as a whole based on context. This weaker drive for central coherence is reflected in both local superiority and global weaknesses (Happé \& Booth, 2008). Local processing refers to featural and detail-focused information processing, whereas global processing is the ability to extract global form and meaning from incoming information (Happé \& Frith, 2006). Alternative explanations like 'enhanced perceptual functioning' (EPF) emphasize enhanced low-level perception leading up to superior local processing without invoking a deficit in global processing (Mottron et al.,

This is the peer reviewed version of the following article:

Maljaars, J., Noens, I., Scholte, E., Verpoorten, R., \& Van Berckelaer-Onnes, I. (2011). Visual local and global processing in low-functioning deaf individuals with and without autism spectrum disorder. Journal of Intellectual Disability Research, 55, 95-105, which has been published in final form at http://onlinelibrary.wiley.com/doi/10.1111/j.1365-2788.2010.01351.x/abstract.

This article may be used for non-commercial purposes in accordance with Wiley Terms and Conditions for self-archiving 
2006; Wang et al., 2007). Nowadays, local and global processing are more and more considered as two independent mechanisms (Happé \& Booth, 2008; Lopez et al., 2008). Despite robust findings of strong local processing in ASD, evidence of impaired global processing is mixed (Happé \& Frith, 2006). However, in many studies it is difficult to disentangle local versus global preferences (Happé \& Booth, 2008).

Local and global processing can be examined in the visual / visuo-spatial and auditory / verbal domain. Preferences in visual perception are often studied in high-functioning individuals with ASD. Despite the relatively high prevalence of intellectual disability (ID) in ASD (Fombonne, 2005; Goin-Kochel et al., 2008), the group of low-functioning individuals with ASD is generally neglected in research. Enhanced local perception in individuals with ASD and ID has been previously determined (Noens \& Van Berckelaer-Onnes, 2008), but the specificity of this enhancement warrants further investigation. Therefore, in the present study visual perception is examined by comparing deaf and hearing individuals with ID with and without ASD. Furthermore, the underlying cognitive style of visual processing in deaf and hearing individuals with ASD is evaluated.

The style of visual processing in individuals with both ASD and ID has been investigated only incidentally. The study of Van Lang et al. (2006) showed that adolescents with ASD and a moderate or mild ID have a weaker drive for central coherence based on two cognitive tasks: Block Design and Child Embedded Figures Test, than IQ-matched adolescents without ASD. However, a recent study in individuals with mild ID found no difference in performance on the Block Design test between participants with high versus low scores on the Social Communication Questionnaire (Harris et al., 2007). These mixed results can possibly be due to differences in level of ID or the criteria used to compose the ASD group.

For individuals with moderate to severe ID, these standard cognitive tasks are often too complicated. In comparison with construction or search tasks, sorting tasks are simpler, because they are unambiguous in nature and no verbal instruction is needed. Even individuals with severe ID are capable of making same versus different judgments or can learn to make these (Serna et al., 1997). The perception of similarities or dissimilarities, as in sorting tasks, involves local and global processing (Förster, 2009). Local processing is helpful in sorting identical materials on perceptual differences, like shape and color (matching). A distinction can be made between matching tasks with meaningless and meaningful materials. Meaningless tasks can be resolved on the basis of local information processing only (shape discrimination). Meaningful tasks can be resolved in the same way, but global information processing can be helpful even though it is not necessary. In contrast, global processing is needed in sorting similar, but non-identical materials on the basis of broader concepts or categories (Noens \& Van Berckelaer-Onnes, 2008). The items of the ComFor (Forerunners in Communication) (Verpoorten et al., 2004, 2008) give the opportunity to study visual perception in individuals with moderate to severe ID, because of the use of different abovementioned sorting tasks. The results of the ComFor study (Noens \& Van Berckelaer-Onnes, 2008) revealed enhanced visual perception in individuals with ASD and ID compared with individuals with ID. In this study, enhancement is a relative term, as enhancement implies superior performance compared with individuals with ID without ASD, thus 'less impaired' and not an enhanced visual perception in comparison with the general population. Item analyses in the ComFor study showed better visual shape discrimination in individuals with ASD and ID compared with individuals with ID, especially in tasks with meaningless materials in which the establishment of meaning is not supportive to sort these materials. These findings are consistent with the WCC account. The present study concerns an extension of the previous ComFor study (Noens \& Van Berckelaer-Onnes, 2008) 
and examines differences in visual perception in ID and ASD and other groups with ID as well. Moreover, the specificity of the WCC account for individuals with ASD and ID compared with other clinical groups is evaluated.

Deafness provides a unique opportunity to examine the influence of auditory deprivation on visual perception (Rettenbach et al., 1999). The question whether intact senses can compensate for a sensory impairment has often been a reason for research (Marschark, 2005). Half of the studies indicate improved visual performance, whereas other studies showed no compensation or even impairment in the remaining senses as well. Age of participants, attention to and localization of the visual stimuli seem to influence the visual performances of the participants (e.g., Bavelier et al., 2006, Dye et al., 2009). Because ID and deafness may have mutual causes, an increased prevalence of deafness in ID can be presumed (Denmark, 1994). The exact prevalence of auditory impairments in individuals with ID is difficult to determine (Evenhuis et al., 2004), but a recent study showed a prevalence of approximately 30\%, which is two times higher than in the general population (Meuwese-Jongejeugd et al., 2006). As a result of the increased risks for both auditory impairments and ASD in ID, comorbidity is also expected to occur frequently. However, the prevalence is unknown, possibly because this group is often neglected in research and also complicated to assess (Vernon \& Rhodes, 2009). Scarce epidemiological studies have shown that auditory problems are more common in ASD and vice versa than in the general population (e.g., Gallaudet Research Institute, 2007; Kielinen et al., 2004; Rosenhall et al., 1999).

The specificity of enhanced visual perception with meaningless materials in individuals with ASD and moderate to severe ID will be evaluated by investigating whether deaf individuals with ID (with and without ASD) also show an enhanced visual perception compared with individuals with ID. The group of individuals with ID is very heterogeneous and therefore it is important to study specific subgroups with ID. Different disorders can bring along different assets and deficits in functioning. Strengths or weaknesses in visual perception in low-functioning deaf individuals with and without ASD will have consequences for treatment. In the present study, visual perception is examined by comparing the results on sorting tasks of four subgroups: individuals with ID, with ID and ASD, with ID and deafness, and with ID, ASD and deafness. As far as we know, this is the first study to explore visual perception in low-functioning deaf individuals with and without ASD.

The following hypotheses will be tested. First, both groups with deafness are expected to show enhanced visual perception in comparison with the ID group, as a result of auditory deprivation. Second, the results on meaningful and meaningless tasks of individuals with ID, ASD and deafness are expected to resemble the results of hearing individuals with ASD and ID, because ASD entails the same underlying cognitive style in both groups. Consistent with results of the previous Comfor study (Noens \& Van Berckelaer-Onnes, 2008) individuals with ASD and ID are expected to show increased scores in comparison with the ID group when the same materials have to be sorted according to perceptual differences, because of strong local perception in ASD. In accordance with the WCC theory these increased scores are mainly driven by enhanced scores on tasks with meaningless materials where global processing is not helpful, but not on tasks with meaningful materials. No differences are expected in sorting tasks with different meaningful materials, because these materials only concern very simple global meanings. Third, in case of deafness without ASD an enhanced score on sorting tasks compared with ID is also to be expected, but not for meaningless or meaningful tasks specifically. Individuals with ASD benefit from relatively superior local processing, whereas individuals with deafness without ASD are expected to have a general enhanced visual perception compared with hearing individuals with ID. 


\section{Method}

\section{Participants}

The total sample consists of 177 children and adults with an adaptive age between 12 and 60 months for the domain of daily living skills measured with the Vineland Screener o-6yrs-NL (Scholte et al., 2008) (see Table 6.1).

Table 6.1 Main characteristics of the four subsamples

\begin{tabular}{lccccccccc}
\hline & \multicolumn{3}{c}{$\begin{array}{c}\text { Adaptive age equivalent } \\
\text { (in months) }\end{array}$} & \multicolumn{2}{c}{$\begin{array}{c}\text { Chronological age } \\
\text { (in months) }\end{array}$} & \multicolumn{2}{c}{$\begin{array}{c}\text { Gender } \\
\text { (in \%) }\end{array}$} \\
Subsamples & $n$ & $M$ & Range & $S D$ & $M$ & Range & $S D$ & Male & Female \\
\hline ID & 68 & 42 & $25-53$ & 7.3 & 181 & $73-580$ & 143.3 & $48 \%$ & $52 \%$ \\
ID+ASD & 72 & 42 & $27-53$ & 6.6 & 171 & $73-605$ & 111.2 & $75 \%$ & $25 \%$ \\
ID+D & 22 & 43 & $26-53$ & 7.6 & 290 & $97-590$ & 124.7 & $68 \%$ & $32 \%$ \\
ID+ASD+D & 15 & 40 & $19-54$ & 10.8 & 248 & $64-381$ & 102.1 & $60 \%$ & $40 \%$ \\
\hline
\end{tabular}

Note. ID = intellectual disability; ASD = autism spectrum disorder; $\mathrm{D}=$ deafness

The total sample comprises four subsamples: (1) individuals with ID $(n=68),(2)$ individuals with ID and ASD ( $n=72)$, (3) individuals with ID and deafness (D) $(n=22)$, and (4) individuals with ID, ASD and $\mathrm{D}(n=15)$. The developmental level for adaptive functioning ranges from 19 to 54 months in groups 3 and 4. Groups 1 and 2 were selected from the data of the ComFor standardization study (Noens et al., 2006). The selection criteria for this study were both a developmental level consistent with groups 3 and 4 and a chronological age above 6 years old. All participants are recruited through institutions where ID $(I \mathrm{C}<70)$ is a condition for admittance and all participants have a moderate to severe ID based on their adaptive level of functioning on the Vineland Screener. ASD was classified according to the criteria of the Diagnostic and Statistical Manual of Mental Disorders, $4^{\text {th }}$ edition, test revision (DSM-IV-TR) [Autistic Disorder or Pervasive Developmental Disorder - Not Otherwise Specified (PDD-NOS)] by qualified professionals (APA, 1994, 2000). Deaf participants suffer from a severe to profound hearing loss (> 7odb loss in the better ear) and have no cochlear implant. Individuals with severe motor impairments and (uncorrected) visual impairments were excluded from all subsamples. The four groups are comparable with respect to adaptive age equivalent $\left(F_{(3,173)}\right.$ $=.43, p=.73)$, but the groups with and without $D$ differ in mean chronological age; the latter are significantly younger $\left(F_{(3,173)}=6.29, p<.01\right)$. There is also a difference in distribution of gender across the subsamples $\left(\chi^{2}{ }_{(3)}=10.83, p<.05\right)$, but no significant differences were found between mean scores of men and women on the main sorting outcomes $(p>.05)$.

\section{Instruments}

Vineland Screener o-6. The Vineland Screener o-6yrs-NL is a questionnaire which can be used to determine the level of adaptive functioning. This screener is adapted from the American Vineland Screeners, versions 0-2;11 and 3-5;11, developed by Sparrow et al. (1993) and recently standardized on a Dutch sample. In this questionnaire with 72 items, a distinction is made between four domains of adaptive functioning: Communication, Daily Living Skills, Socialization and Motor Skills (Scholte et al., 2008). In low-functioning individuals with ASD, the level of adaptive functioning is fairly comparable to the level of intelligence (Bölte \& Poustka, 2002; Liss et al., 2001). Research with individuals with ASD and ID indicated the domain of Daily Living Skills as the best indicator of 
general IQ (Bölte \& Poustka, 2002). Therefore, the adaptive age equivalent for the domain of Daily Living Skills was used to compare the developmental level of the four subsamples. The psychometric properties of reliability and validity for the Dutch version of the Vineland Screener are good (Scholte et al., 2008).

Sorting tasks. Sorting was first trained by completing different assembling tasks. The scores on the assembling tasks were not used to measure visual perception. Only participants who were able to complete the six assembling tasks were assessed by using the sorting tasks. The participants who failed on these items did not have the basic prerequisites for sorting. From the original sample $(N=$ 200), 23 participants made one or more mistakes during the assembling tasks, as they were too low functioning or unable to manipulate the materials adequately because of motor problems.

A selection of 18 different sorting tasks derived from the ComFor (Verpoorten et al., 2004, 2008) was used to measure visual perception (see Table 6.2).

Table 6.2 Short description of the sorting tasks

\begin{tabular}{|c|c|c|}
\hline Tasks & Item & Description \\
\hline \multicolumn{3}{|c|}{ Assembling: prerequisite for sorting } \\
\hline \multirow[t]{6}{*}{ Objects } & 1 & Assembling balls in a closed box \\
\hline & 2 & Assembling blocks in a closed box \\
\hline & 3 & Assembling rings in a closed box \\
\hline & 4 & Assembling rings and balls in closed boxes \\
\hline & 5 & Assembling blocks, balls and rings in closed boxes \\
\hline & 6 & Assembling blocks and balls in closed boxes, spoons in open box \\
\hline \multicolumn{3}{|c|}{$\begin{array}{l}\text { 1. Sorting meaningful identical materials } \\
\rightarrow \text { local information processing is satisfactory, but global information processing can be helpful }\end{array}$} \\
\hline \multirow[t]{3}{*}{ 1a. Objects } & 1 & Sorting identical checkers, screws, little sticks \\
\hline & 2 & Sorting identical spoons, knives, forks \\
\hline & 3 & Sorting identical dessert, tea, soup spoons \\
\hline \multirow[t]{3}{*}{ 1b. Pictures } & 1 & Sorting identical black / white photographs of combing hair, eating, brushing teeth \\
\hline & 2 & Sorting identical black / white photographs of one person reading, writing, drinking \\
\hline & 3 & Sorting identical line drawings of combing hair, eating, brushing teeth \\
\hline \multicolumn{3}{|c|}{$\begin{array}{l}\text { 2. Sorting meaningless identical materials } \\
\rightarrow \text { local information processing only }\end{array}$} \\
\hline \multirow[t]{3}{*}{ 2a. Objects } & 1 & Sorting identical blue, yellow, red checkers \\
\hline & 2 & Sorting identical small circles, big circles, ovals \\
\hline & 3 & Sorting identical cubes, thin blocks, wide blocks \\
\hline \multirow[t]{3}{*}{ 2b. Pictures } & 1 & Sorting identical letters $\mathrm{E}, \mathrm{F}, \mathrm{L}$ \\
\hline & 2 & Sorting identical numbers $25,26,29$ \\
\hline & 3 & Sorting identical words bed, bad, kam (English: bed, bath, comb) \\
\hline \multicolumn{3}{|c|}{$\begin{array}{l}\text { 3. Sorting meaningful different materials } \\
\rightarrow \text { global information processing, local information processing is contra productive }\end{array}$} \\
\hline \multirow[t]{3}{*}{ 3a. Objects } & 1 & Sorting different balls, dolls, cars \\
\hline & 2 & Sorting different spoons, knives, forks \\
\hline & 3 & Sorting functionally related objects: toothbrush-toothpaste, pencil-notebook, fork-plate \\
\hline \multirow[t]{3}{*}{ 3b. Pictures } & 1 & Sorting photographs of woman, ball, car in different situations \\
\hline & 2 & Sorting photographs of comb, toothbrush, spoon in different situations \\
\hline & 3 & $\begin{array}{l}\text { Sorting photographs of writing, combing hair, drinking in complex context with pen, } \\
\text { comb, cup }\end{array}$ \\
\hline
\end{tabular}

Because there are no common used tasks or instruments to evaluate visual perception and WCC in individuals with moderate to severe ID, the tasks used in the ComFor offer a good alternative. As mentioned in the introduction, sorting tasks are very suitable to measure visual perception in individuals with moderate to severe ID. To complete a sorting task, the participant has to sort nine objects or pictures correctly in three boxes with one example in each of the boxes. 
The sorting tasks were divided in three categories with each six items: meaningful identical materials, meaningless identical materials and meaningful different materials. Within these categories, a distinction is made between the subcategories objects and pictures. For each subcategory of sorting tasks, only the three most difficult items were selected. In this way, nondiscriminating items and items which were not clearly meaningful or meaningless were not used in this study. In the first two categories of sorting tasks, the identical objects or pictures have to be matched according to shape, color, matter and size. Thus, these tasks can be resolved on the basis of concrete, literally perceptible features. A further distinction can be made between tasks with meaningless and meaningful materials. Meaningless materials include for example black abstract shapes, numbers or small words. For most individuals with moderate to severe ID items with numbers and words are meaningless, because they are not familiar with written language and are not able to read, whereas examples of meaningful materials are pictures of utensils or simple actions, like drinking or combing hair. Meaningless and meaningful items can be resolved on the basis of local information processing only (e.g., shape discrimination), global information processing can only be helpful in meaningful items. In the third category, non-identical objects or pictures have to be sorted on the basis of sense-making beyond the concrete, literally perceptible features. To solve these tasks global information processing is indispensable, but it only concerns primary and functional meanings. In sorting different meaningful materials, a focus on local information is usually contra productive (Noens et al., 2006). The reliability of the measurements of the various categories is .76 and above (Cronbach's alpha).

\section{Procedure}

Participants were recruited from several institutions for persons with ID and/or hearing impairments in the Netherlands and Flanders (Belgium). First, informed consent was obtained from the parents. The caregivers of the participants in the institutions were asked to complete the Vineland Screener o-6yrs-NL. Subsequently, all participants were assessed using the sorting tasks. Assessments were carried out in the participant's institution by one of the authors (JM) or trained master's students of the Leiden University. The administrations were all videotaped to allow inspection of assessment procedure and consensus scoring.

\section{Data analyses}

SPSS 16.0 was used to conduct the analyses. First, performances of the four groups on the total score for the sorting tasks were examined to determine a possibly enhanced visual perception in the subsamples with ASD and/or D in comparison with the subsample with ID. The scores were calculated by summing the item scores. Second, differences between the subgroups on meaningful and meaningless items were analyzed to evaluate differences in visual perception between the four subgroups and the underlying cognitive style in the subgroups with ASD. Differences in mean scores between the four groups were evaluated by conducting several analyses of covariance (ANCOVA) in combination with planned comparisons with two contrasts. The first contrast concerned the comparisons between the subsamples ID vs. ID+ASD, ID vs. ID+D and ID vs. ID+ASD+D, the second contrast between the subsamples ID+ASD vs. ID+D and ID+ASD+D vs. ID+D. Given the differences in mean chronological age between the groups and the variance in adaptive age equivalents (Daily Living Skills) within the groups, these variables were included as covariates in the General Linear Model. The validation study of the ComFor revealed a significant relation between ComFor scores and the domain of Daily Living Skills (Noens et al., 2006). Gender was not used in the model, 
because it was not significantly related to the outcome variables ( $p>.05$ for all analyses). Furthermore, the differences between tasks with objects or pictures were studied in more detail. $A$ more stringent significance level of .01 was established throughout all analyses, because of the relatively large number of analyses of variance conducted.

\section{Results}

For all sorting tasks, the subsample with individuals with three classifications (ID+ASD+D) obtained the highest mean scores compared with the other subsamples. Furthermore, the subsamples with ID+D and ID+ASD performed also better than the subsample with ID only (see Table 6.3).

Table 6.3 Description of adjusted means of the ComFor-scores

\begin{tabular}{|c|c|c|c|c|c|c|c|c|c|}
\hline \multirow[b]{3}{*}{ Subsamples } & \multirow[b]{3}{*}{$n$} & \multicolumn{6}{|c|}{ Sorting identical materials } & \multirow{2}{*}{\multicolumn{2}{|c|}{$\begin{array}{c}\text { Sorting different materials } \\
\text { Meaningful } \\
\text { (6 items) }\end{array}$}} \\
\hline & & \multicolumn{2}{|c|}{$\begin{array}{c}\text { Total } \\
\text { (12 items) }\end{array}$} & \multicolumn{2}{|c|}{$\begin{array}{l}\text { Meaningful } \\
\text { (6 items) }\end{array}$} & \multicolumn{2}{|c|}{$\begin{array}{l}\text { Meaningless } \\
\text { (6 items) }\end{array}$} & & \\
\hline & & $M$ & $S D$ & $M$ & $S D$ & $M$ & $S D$ & $M$ & $S D$ \\
\hline ID & 68 & 5.2 & .37 & 3.0 & .19 & 2.1 & .20 & 1.7 & .22 \\
\hline ID+ASD & 72 & $7.2^{b}$ & .36 & $3.7^{\mathrm{a}}$ & .19 & $3.5^{b}$ & .20 & $2.3^{\mathrm{a}}$ & .22 \\
\hline ID+D & 22 & $6.4^{\mathrm{a}}$ & .67 & $3.5^{\mathrm{a}}$ & .35 & $2.9^{\mathrm{a}}$ & .37 & $1.9^{\mathrm{a}}$ & .41 \\
\hline$I D+A S D+D$ & 15 & $8.0^{\mathrm{b}}$ & .79 & $4.2^{\mathrm{a}}$ & .41 & $3.7^{b}$ & .43 & $2.1^{\mathrm{a}}$ & .48 \\
\hline
\end{tabular}

a no difference with ID; ${ }^{\mathrm{b}}$ significantly higher than ID, no difference with ID+D

The analyses of covariance revealed that both covariates, adaptive and chronological age, are significantly related to the results on the different sorting tasks $(p<.01)$, with the exception of chronological age in relation to the sorting tasks concerning different meaningful materials $(p>.01)$. There was also a significant effect of diagnosis on the total score for sorting identical materials $\left(F_{(3,166)}=6.87, p<.001\right)$. Planned contrasts revealed that the scores were significantly higher for the subsamples with ID+ASD and ID+ASD+D compared with ID $(p<.01)$. The mean score of the subsample ID+D was not significantly different from the higher scores in the ID+ASD and ID+ASD+D groups, or from the lower score in the ID group $(p>$.01). This group seems to take a position in the middle of these groups. The differences between the groups on tasks with identical materials were significant for meaningless tasks $\left(F_{(3,166)}=9.44, p<.001\right)$ and planned contrasts showed the same significant differences as mentioned above for sorting identical materials $(p<.001)$. No significant differences were found for meaningful tasks with identical materials $\left(F_{(3,166)}=3.51, p>.01\right)$ or different materials $\left(F_{(3,166)}=1.04, p>.01\right)$.

\section{Discussion}

The ComFor study (Noens \& Van Berckelaer-Onnes, 2008) has indicated that individuals with ID and ASD show enhanced visual local processing compared with individuals with ID only. Item analyses revealed that this enhancement can be explained by WCC in ASD. The main objective of this study was to examine the performances on sorting tasks of the ComFor; first, to determine whether an enhanced visual perception is also present in low-functioning deaf individuals with and without ASD, and second, to evaluate the underlying cognitive style in the two subgroups with ASD. 
The results from this study showed that in addition to individuals with ID and ASD, also deaf individuals with ID and ASD obtained a higher total score on the sorting tasks compared with individuals with ID, but not when compared with individuals with ID and deafness. These findings indicate enhanced visual perception in low-functioning individuals with ASD with and without deafness in comparison with individuals with the same adaptive level of functioning without ASD. The scores of the deaf groups indicate that visual perception is also enhanced in these groups, but the enhancement is less clear in the deaf group without ASD. Other studies into deafness mainly found differences in visual processing when visual stimuli were presented in the peripheral visual field (e.g., Bavelier et al., 2006; Dye et al., 2009; Reynolds, 1993), whereas the sorting tasks concerned both stimuli in the central and the peripheral visual field. One of the main reasons why some studies found no differences in or even worse visual sensitivity in the central field in deaf individuals concerns their increased visual distractibility by irrelevant peripheral stimuli (Dye et al., 2008). Hence, the Comfor was administered by one person in a separate, quiet and visually predictable environment allowing participants to focus upon the tasks at hand. However, all mentioned studies into visual perception and deafness are difficult to compare with this study because in all other studies only participants with cognitive abilities in the mean or even higher functioning range took part.

The evaluation of the total performance on the sorting tasks does not address the question which underlying cognitive style is responsible for the enhanced performances in the different groups with ASD. Therefore, differences in comparisons between groups for meaningless and meaningful items were taken into account. The group with ID and ASD obtained higher scores on meaningless items only compared with the ID group, which indicates enhanced local information processing alongside reduced global processing (Noens \& Van Berckelaer-Onnes, 2008). All sorting tasks concerning identical materials could be sorted according to shape. To solve the meaningless items, global information processing and sense-making beyond literally perceptible features cannot offer any support and the tasks have to be sorted on the base of local information processing. Results could not be explained by good shape discrimination alone, otherwise the differences would be found in both meaningful and meaningless sorting tasks. Therefore, the findings of the analyses for meaningful and meaningless tasks support the hypothesis of the WCC theory and are not consistent with the EPF hypothesis. In contrary to the WCC theory, the EPF predicts higher scores on both meaningless and meaningful items, because of superior local processing which becomes more evident in the tasks concerning the most difficult shape discriminations regardless of the content of the materials.

Despite the smaller groups of deaf individuals, also a significantly higher score on meaningless tasks and not on meaningful tasks was found for deaf individuals with ASD, whereas no differences were found on meaningless or meaningful tasks for deaf individuals without ASD. As expected, only the results of the deaf individuals with ID and ASD are in line with the results of the hearing individuals with ID and ASD. These results indicate the same underlying cognitive style in both deaf and hearing individuals with ASD.

In summary, like in individuals with ID and ASD, enhanced performance on the sorting tasks in deaf individuals with ID and ASD compared with individuals with ID can be understood within the framework of the central coherence theory, whereas in deaf individuals with ID without ASD the enhancement might be due to a more generally enhanced visual perception. WCC in both groups with ASD is reflected in superior performance on meaningless sorting tasks where the ability to 
process local information is advantageous, because the groups without ASD cannot benefit from a more global processing style.

In the study of Van Lang et al. (2006) also indications for a weaker drive for central coherence in individuals with ID and ASD were found in the performances on the Block Design Test and the Child Embedded Figures Test, whereas Harris et al. (2007) failed to find the same results on the Block Design Test. The most important difference between these two studies is the level of functioning of the participants. Both studies focused on participants with ID, but the former study included mild and moderate ID while the latter only addressed mild ID or even borderline intellectual functioning. Based on the results of before-mentioned studies, it can be hypothesized that a WCC in the visual or visuo-spatial domain becomes clearer in performances on less complex tasks as the level of cognitive functioning decreases. To study WCC in the visual domain among high-functioning individuals with ASD even more complex visual tasks which require global perception of complex symbolic meanings are necessary to reveal this processing style (see for example Nakahachi et al., 2008). In addition, all studies into WCC in individuals with ASD and ID only examined the style of processing in the visual / visuo-spatial domain. Studies into the auditory / verbal domain are difficult to carry out, because low-functioning individuals often lack the verbal abilities to perform the tasks.

Results of the present study have to be interpreted in the context of the study's limitations. The first shortcoming is related to the instrument used in this study. Several sorting tasks derived from the ComFor are applied to measure visual perception, whereas the main purpose of this clinical instrument concerns the assessment for the indication of augmentative communication based on perception and level of sense-making (Verpoorten et al., 2004, 2008). However, the sorting procedure used in this instrument is very suitable to measure visual perception in low-functioning individuals. Furthermore, there are differences in size and chronological age between the subsamples. The groups with deafness are smaller and significantly older than the hearing groups with ID and/or ASD. Therefore, results have to be interpreted with caution, because ANCOVA is less robust to violations of homogeneity of variance when group sizes are unequal. Recruiting (young) deaf individuals with ID and ASD turned out to be very difficult, also aggravated by the strict criteria we had to use for deafness and ASD in ID. Only individuals who passed a hearing test and had an official DSM-classification for autistic disorder or PDD-NOS were considered for participation, as we lacked a suitable (screening) instrument to classify ASD in deafness (Vernon \& Rhodes, 2009). Although ASD can be diagnosed in deaf individuals, it is much later recognized than in the hearing population, often not before adolescence (Roper et al., 2003). A broader international collaboration is necessary to improve research into individuals with ID, ASD and deafness. Finally, a control group of individuals with ID is used. This group can be characterized as a very heterogeneous group, but the common characteristics are no hearing loss or ASD. To reveal strengths and weaknesses in specific conditions with ID, the use of a heterogeneous control group of ID is much more preferable than a typical or specific control group like Down syndrome (Hodapp \& Dykens, 2001). To ensure comparability between the subsamples, all groups were definitely matched for level of adaptive functioning according to the criteria for matching (Mervis \& Klein-Tasman, 2004).

Despite these limitations, the current findings do have implications for our understanding of deafness and ASD in ID, and the processes involved in visual perception and sense-making. A clearer understanding of the nature of mechanisms underlying problems in perception and sense-making has the potential to improve interventions. WCC is not only present in hearing, but also in deaf individuals with ID and ASD. This study shows that ASD and deafness do not only result in deficits, but can bring along assets, like a strong local visual perception, in functioning as well. Because ASD 
and deafness have communication problems in common, the presence of both conditions in one person can lead to more severe communication problems, therefore treatment becomes more complex (Vernon \& Rhodes, 2009). The understanding and use of sign language is not always achievable in low-functioning deaf individuals with ASD. Therefore, other communication strategies have to be applied to maximize communication. In clinical practice, it is important to realize that individuals with associated disabilities may benefit from other augmentative communication strategies compared with individuals with ID only. Strengths in visual perception and abilities in sense-making can be used to offer the best suitable intervention to support communication.

\section{References}

American Psychiatric Association [APA] (1994). Diagnostic and Statistical Manual of Mental Disorders (4th ed.). Washington, DC: APA.

American Psychiatric Association [APA] (2000). Diagnostic and Statistical Manual of Mental Disorders (4th ed., text revision). Washington, DC: APA.

Bavelier, D., Dye, M. W. G., \& Hauser, P. C. (2006). Do deaf individuals see better? Trends in Cognitive Sciences, 10, 512-518.

Bölte, S. \& Poustka, F. (2002). The relation between general cognitive level and adaptive behavior domains in individuals with autism with and without co-morbid mental retardation. Child Psychiatry and Human Development, 33, 165-172.

Denmark, J. C. (1994). Deafness and mental health. London, UK: Jessica Kingsley.

Dye, M. W. G., Hauser, P. C., \& Bavelier, D. (2008). Visual attention in deaf children and adults: implications for learning environments. In M. Marschark \& P. C. Hauser (Eds.), Deaf cognition: Foundations and outcomes (pp. 250-263). New York, NY: Oxford University Press.

Dye, M. W. G., Hauser, P. C. \& Bavelier, D. (2009). Is visual selective attention in deaf individuals enhanced or deficient? The case of the useful field of view. PLoSONE 4(5): e5640. doi:10.1371/journal.pone.0005640.

Evenhuis, H., Van Splunder, J., Vink, M., Weerdenburg, C., Van Zanten, B., \& Stilma, J. (2004). Obstacles in large-scale epidemiological assessment of sensory impairments in a Dutch population with intellectual disabilities. Journal of Intellectual Disability Research, 48, 708-718.

Fombonne, E. (2005). Epidemiological studies of pervasive developmental disorders. In F. Volkmar, R. Paul, A. Klin, \& D. J. Cohen (Eds.). Handbook of autism and pervasive developmental disorders (3rd edn) (pp. 42-69). New York, NY: Wiley.

Förster, J. (2009). Relations between perceptual and conceptual scope: how global versus local processing fits a focus on similarity versus dissimilarity. Journal of Experimental Psychology: General, 138, 88-111.

Frith, U. (1989). Autism. Explaining the enigma. Oxford, UK: Blackwell.

Gallaudet Research Institute [GRI] (2007). Regional and national data from 2006-2007: annual survey of deaf and hard of hearing children and youth. Washington, DC: Gallaudet University.

Goin-Kochel, R. P., Peters S. U., \& Treadwell-Deering, D. (2008). Parental reports on the prevalence of co-occurring intellectual disability among children with autism spectrum disorders. Research in Autism Spectrum Disorders, 2, $546-556$.

Happé, F. G. E., \& Booth, R. D. L. (2008). The power of the positive: revisiting weak coherence in autism spectrum disorders. The Quarterly Journal of Experimental Psychology, 61, 50-63.

Happé, F., \& Frith, U. (2006). The weak coherence account: detail-focused cognitive style in autism spectrum disorders. Journal of Autism and Developmental Disorders, 36, 5-25.

Harris, J. M., Best, C. S., Moffat, V. J., Spencer, M. D., Philip, R. C. M., Power, M. J., \& Johnstone, E. C. (2007). Autistic traits and cognitive performance in young people with mild intellectual impairment. Journal of Autism and Developmental Disorders, 38, 1241-1249.

Hodapp, R. M., \& Dykens, E. M. (2001). Strengthening behavioral research on genetic mental retardation syndromes. American Journal on Mental Retardation, 106, 4-15.

Kielinen, M., Rantala, H., Timonen, E., Linna, S. L., \& Moilanen, I. (2004). Associated medical disorders and disabilities in children with autistic disorder. Autism, 8, 49-60.

Liss, M., Harel, B., Fein, D., Allen, D., Dunn, M., Feinstein, C., ... \& Rapin, I. (2001). Predictors and correlates of adaptive functioning in children with developmental disorders. Journal of Autism and Developmental Disorders, 31, 219-230.

Lopez, B., Leekam, S. R., \& Arts, G. R. J. (2008). How central is central coherence? Preliminary evidence on the link between conceptual and perceptual processing in children with autism. Autism, 12, 159-171.

Marschark, M. (2005). Psychological development of deaf children. New York, NY: Oxford University Press.

Mervis, C. B., \& Klein-Tasman, B. P. (2004). Methodological issues in group-matching designs: $\alpha$ levels for control variable comparisons and measurement characteristics of control and target variables. Journal of Autism and Developmental Disorders, 34, 7-17. 
Meuwese-Jongejeugd, A., Vink, M., Van Zanten, B., Verschuure, H., Bernsen, R., \& Evenhuis, H. (2006). Prevalence of hearing impairment in 1,598 adults with an intellectual disability: cross-sectional population-based study. International Journal of Audiology, 45, 660-669.

Mottron, L., Dawson, M., Soulières, I., Hubert, B., \& Burack, J. (2006). Enhanced perceptual functioning in autism: an update and eight principles of autistic perception. Journal of Autism and Developmental Disorders, 36, 27-43.

Nakahachi, T., Yamashita, K., Iwase, M., Ishigami, W., Tanaka, C., Toyonaga, K., ... \& Takeda, M. (2008). Disturbed holistic processing in autism spectrum disorders verified by two cognitive tasks requiring perception of complex visual stimuli. Psychiatry Research, 156, 330-338.

Noens, I. L. J., \& Van Berckelaer-Onnes, I. A. (2008). The central coherence account of autism revisited: evidence from the ComFor study. Research in Autism Spectrum Disorders, 2, 209-222.

Noens, I., Van Berckelaer-Onnes, I., Verpoorten, R., \& Van Duijn, G. (2006). The ComFor: an instrument for the indication of augmentative communication in people with autism and intellectual disability. Journal of Intellectual Disability Research, 50, 621-632.

Rettenbach, R., Diller, G., \& Sireteanu, R. (1999). Do deaf people see better? Texture segmentation and visual search compensate in adult but not in juvenile subjects. Journal of Cognitive Neuroscience, 11, 560-583.

Reynolds, H. N. (1993). Effects of foveal stimulation on peripheral visual processing and laterality in deaf and hearing subjects. The American Journal of Psychology, 106, 523-540.

Roper, L., Arnold, P., \& Monteiro, B. (2003). Co-occurrence of autism and deafness. Autism, 7, 245-253.

Rosenhall, U., Nordin, V., Sandström, M., Ahlsén, G., \& Gilberg, G. (1999). Autism and hearing loss. Journal of Autism and Developmental Disorders, 29, 349-357.

Scholte, E., Van Duijn, G., Dijkxhoorn, Y., Noens, I., \& Van Berckelaer-Onnes, I. (2008). Handleiding Vineland Screener o-6 [Manual Vineland Screener o-6yrs-NL]. Leiden, NL: PITS.

Serna, R. W., Dube, W. V., \& Mcllvane, W. J. (1997). Assessing same/different judgments in individuals with severe intellectual disabilities: A status report. Research in Developmental Disabilities, 18, 343-368.

Sparrow, S. S., Carter, A. S., \& Cicchetti, D. (1993). Vineland Screener. overview, reliability, validity, administration and scoring. New Haven, CT: Yale University.

Van Lang, N. D. J., Bouma, A., Sytema, S., Kraijer, D. W., \& Minderaa, R. B. (2006). A comparison of central coherence skills between adolescents with an intellectual disability with and without comorbid autism spectrum disorder. Research in Developmental Disabilities, 27, 217-226.

Vernon, M., \& Rhodes, A. (2009). Deafness and autistic spectrum disorders. American Annals of the Deaf, 154, 5-14.

Verpoorten, R., Noens, I., \& Van Berckelaer-Onnes, I. (2004). De ComVoor-Voorlopers in communicatie. Handleiding. [The ComFor - Forerunners in communication. Manual]. Leiden, NL: PITS.

Verpoorten, R., Noens, I. \& Van Berckelaer-Onnes, I. (2008). The ComFor - Forerunners in communication. Manual. Leiden, NL: PITS. 